Research Article

\title{
Optimization of the PNG Law for a Dual-Spin Mortar with Fixed Canards
}

\author{
Pengfei Liu $\left(\mathbb{D},{ }^{1}\right.$ Hongsong Cao $\mathbb{D}^{1},{ }^{1}$ Shunshan Feng, ${ }^{2}$ Hengzhu Liu, ${ }^{1}$ and Lifei Cao ${ }^{1}$ \\ ${ }^{1}$ College of Mechatronics Engineering, North University of China, Taiyuan 030051, China \\ ${ }^{2}$ State Key Laboratory of Explosion Science and Technology, Beijing Institute of Technology, Beijing 100081, China
}

Correspondence should be addressed to Hongsong Cao; chs@nuc.edu.cn

Received 6 June 2021; Revised 9 August 2021; Accepted 22 October 2021; Published 2 November 2021

Academic Editor: Xiaodong Sun

Copyright (c) 2021 Pengfei Liu et al. This is an open access article distributed under the Creative Commons Attribution License, which permits unrestricted use, distribution, and reproduction in any medium, provided the original work is properly cited.

The limited instantaneous overload available and the curved trajectory lead to adaptivity problems for the proportional navigation guidance (PNG) of a guided mortar with a fixed-canard trajectory correction fuze. In this paper, the optimization of a PNG law with gravity compensation is established. Instead of using the traditional empirical method, the selection of the proportional navigation constants is formulated as an optimization problem, which is solved using an intelligent optimization algorithm. Two optimization schemes are proposed for constructing corresponding optimization models. In schemes 1 and 2, the sum squared error between the impact point and target and the circular error probability (CEP), respectively, are taken as the objective function. Monte Carlo simulations are conducted to verify the effectiveness of the two optimization schemes, and their guidance performance is compared through trajectory simulations. The simulation results show that the impact point dispersion can be efficiently reduced under both proposed schemes. Scheme 2 achieves a lower CEP, which is approximately $2.9 \mathrm{~m}$ and 2.4 times smaller than that achieved by scheme 1 . Moreover, the mean impact point is closer to the target.

\section{Introduction}

With the increasing operational requirements regarding mortar delivery accuracy and maneuverability as well as collateral damage and round expenditure, trajectory correction mortars have received significant research attention [1-7].

As one of the key technologies for guided ammunition, guidance methods are always a focus of research in this field $[2,8]$. The proportional navigation guidance (PNG) law has computational simplicity, robustness, and implementability and has been widely used in various kinds of trajectory correction projectiles [3]. However, flight at a subsonic velocity induces a small aerodynamic control force. The limited control force and low velocity result in the availability of insufficient instantaneous overloads. Moreover, for a guided mortar with a dual-spin configuration with forward-mounted fixed canards, the fixed cant angle causes the magnitude of the correction force to be uncontrollable. Meanwhile, the required overload due to the curved trajectories of such mortar projectiles is large and may exceed the available instantaneous overloads; as a result, such a projectile may not approach the line of sight at the angular rate specified in conventional PNG.

Much research has been devoted to methods to reduce the required overload and improve the firing accuracy. Guo considered the lateral deviation with an advanced proportional guidance law in the ascending segments and conducted comprehensive correction with conventional PNG in the descending segments [2]. Cheng studied a modified proportional navigation algorithm with two-dimensional correction based on fixed canards by making use of the zeroeffort-miss concept, in which the target location is chosen as the control variable [9]. Zhang took the horizontal velocity and position as the control variables to construct a new adaptive proportional-derivative guidance law for a guided mortar projectile [10]. It is well known that the proportional navigation constants are the key factors for the PNG law [11]. However, in the above-mentioned works, the proportional navigation constants were determined 
experimentally and have relatively low efficiency and strong subjectivity, and the entire parameter spaces cannot be comprehensively evaluated.

In this paper, a novel idea is introduced for the determination of the proportional navigation constants. The intelligent optimization algorithm is used to determine the proportional navigation constants for a PNG law with gravity compensation. Two optimization schemes are proposed for the construction of corresponding optimization models, in which either the sum squared error between the impact point and target or the circular error probability (CEP) is taken as the objective function. Several simulations are conducted to compare the performance of these two schemes. In the simulation model, a dual-spin mortar projectile with fixed canards is selected as the test bed, and the target is set to be nonmaneuvering. A Global Positioning System (GPS) receiver is used to measure the position of the mortar projectile, and a three-axis magnetometer is used to obtain the roll angular rate and roll angle.

The present paper is organized as follows: Section 2 outlines the nonlinear projectile flight dynamic model and its properties. Section 3 presents the PNG laws for the vertical and horizontal planes and the gravity compensation formulation. Additionally, two optimization schemes are proposed for constructing corresponding optimization problems. Section 4 describes the simulation results. Finally, the conclusions are presented in Section 5.

\section{Projectile Flight Dynamic Model}

In this study, a standard six-degree-of-freedom (6-DOF) model is used to simulate the flight trajectories of the mortar projectile. As illustrated in Figure 1, the two reference frames are used to develop this model. The Earth coordinate system (subscript $E$ ) is used as the inertial frame, and the body fixedplane coordinate system (subscript BFP) is used as the body frame [12]. The six degrees of freedom of the model consist of the inertial position components of the projectile mass center $(x, y, z)$ in the inertial frame and the standard aerospace sequence Euler angles $(\psi, \theta, \phi)$. The equations of motion for the projectile flight dynamic model are given as follows $[12,13]$ :

$$
\begin{aligned}
& {\left[\begin{array}{c}
\dot{x}_{E} \\
\dot{y}_{E} \\
\dot{z}_{E}
\end{array}\right]=\left[\begin{array}{ccc}
\cos \theta \cos \psi & -\sin \psi & \sin \theta \cos \psi \\
\cos \theta \sin \psi & \cos \psi & \sin \theta \sin \psi \\
-\sin \theta & 0 & \cos \theta
\end{array}\right]\left[\begin{array}{c}
u \\
v \\
w
\end{array}\right],} \\
& {\left[\begin{array}{c}
\dot{\phi} \\
\dot{\theta} \\
\dot{\psi}
\end{array}\right]=\left[\begin{array}{ccc}
1 & 0 & \tan \theta \\
0 & 1 & 0 \\
0 & 0 & \frac{1}{\cos \theta}
\end{array}\right]\left[\begin{array}{c}
p \\
q \\
r
\end{array}\right],} \\
& {\left[\begin{array}{c}
\dot{u} \\
\dot{v} \\
\dot{w}
\end{array}\right]=\frac{1}{m}\left[\begin{array}{c}
X \\
Y \\
Z
\end{array}\right]-\left[\begin{array}{ccc}
0 & -r & q \\
r & 0 & r \tan \theta \\
-q & -r \tan \theta & 0
\end{array}\right]\left[\begin{array}{c}
\dot{u} \\
\dot{v} \\
\dot{w}
\end{array}\right],}
\end{aligned}
$$

$$
\left[\begin{array}{c}
\dot{p} \\
\dot{q} \\
\dot{r}
\end{array}\right]=\mathbf{I}^{-1}\left(\left[\begin{array}{c}
L \\
M \\
N
\end{array}\right]-\left[\begin{array}{ccc}
0 & -r & q \\
r & 0 & r \tan \theta \\
-q & -r \tan \theta & 0
\end{array}\right] \mathbf{I}\left[\begin{array}{l}
p \\
q \\
r
\end{array}\right]\right) .
$$

In (3) and (4), the total applied force and moment components include contributions from the gravity $(\mathrm{G})$, the aerodynamic force $(\mathrm{A})$, the Magnus aerodynamic force $(M)$, and the canard control force $(\mathrm{C})$, as shown in the following equations:

$$
\begin{aligned}
& {\left[\begin{array}{c}
X \\
Y \\
Z
\end{array}\right]=\left[\begin{array}{c}
X_{G} \\
Y_{G} \\
Z_{G}
\end{array}\right]+\left[\begin{array}{c}
X_{A} \\
Y_{A} \\
Z_{A}
\end{array}\right]+\left[\begin{array}{c}
X_{M} \\
Y_{M} \\
Z_{M}
\end{array}\right]+\left[\begin{array}{c}
X_{C} \\
Y_{C} \\
Z_{C}
\end{array}\right],} \\
& {\left[\begin{array}{c}
L \\
M \\
N
\end{array}\right]=\left[\begin{array}{c}
L_{A} \\
M_{A} \\
N_{A}
\end{array}\right]+\left[\begin{array}{c}
L_{M} \\
M_{M} \\
N_{N}
\end{array}\right]+\left[\begin{array}{c}
L_{U A} \\
M_{U A} \\
N_{U A}
\end{array}\right]+\left[\begin{array}{c}
L_{C} \\
M_{C} \\
N_{C}
\end{array}\right] .}
\end{aligned}
$$

The canard control force $(\mathrm{C})$ and moment on the projectile can be presented as follows:

$$
\left[\begin{array}{c}
X_{C} \\
Y_{C} \\
Z_{C}
\end{array}\right]=\frac{1}{2} \rho V_{C G}^{2} S\left[\begin{array}{c}
C_{C C A} \\
C_{C C Y \delta}\left(\delta_{r}-\beta\right) \\
-C_{C C N \delta}\left(\delta_{q}+\alpha\right)
\end{array}\right],
$$

$$
\left[\begin{array}{c}
L_{C} \\
M_{C} \\
N_{C}
\end{array}\right]=\left[\begin{array}{c}
0 \\
-Y_{C} \\
Z_{C}
\end{array}\right] x_{C c p}=Q A x_{C c p}\left[\begin{array}{c}
0 \\
C_{C C N \delta}\left(\delta_{q}+\alpha\right) \\
C_{C C Y \delta}\left(\delta_{r}-\beta\right)
\end{array}\right],
$$

where $\delta_{r}$ and $\delta_{q}$ are virtual signals that depend on the nose angular orientation $\phi_{N}$ and on the applied canard pair deflections $\delta_{Y}$ and $\delta_{Z}$ via the following projection equation:

$$
\begin{aligned}
& {\left[\begin{array}{l}
0 \\
\delta_{r} \\
\delta_{q}
\end{array}\right]=\left[\begin{array}{ccc}
1 & 0 & 0 \\
0 & \cos \phi_{N} & \sin \phi_{N} \\
0 & -\sin \phi_{N} & \cos \phi_{N}
\end{array}\right]\left[\begin{array}{c}
0 \\
\delta_{Y} \\
\delta_{Z}
\end{array}\right]} \\
& =\left[\begin{array}{c}
0 \\
\delta_{Y} \cos \phi_{N}+\delta_{Z} \sin \phi_{N} \\
-\delta_{Y} \sin \phi_{N}+\delta_{Z} \cos \phi_{N}
\end{array}\right],
\end{aligned}
$$

where the projection matrix represents the transformation matrix from the coordinate frame linked to the canards to the BFP frame; more details can be found in [14]. $C_{C C N \delta}$ and $C_{C C Y \delta}$ are the canard force aerodynamic coefficients, with $C_{C C N \delta}=C_{C C Y \delta} \cdot x_{C c p}$ is the center of pressure of the canards from the moment reference center of the body. The terms $C_{C C N \delta}, C_{C C Y \delta}$, and $x_{C c p}$ depend on the local Mach number.

In this paper, the fourth-order Runge-Kutta algorithm is selected to solve the above model. In the present analysis, the actuators were not modeled in detail; rather, external forces and moments were specified and applied on the nose of the guided projectile. The aerodynamics of guided and unguided projectiles were considered identical. External control forces were applied along the $O y_{F P}$ and $O z_{F P}$ axes of the fixed- 
plane coordinate system. Additional equations of the projectile flight dynamic model and more details can be found in $[12,15]$.

\section{Optimization of the PNG Law}

PNG is a method of guidance in which the angular rate of the projectile velocity vector, $\dot{\sigma}$, is proportional to the line-ofsight (LOS) angular rate, $\dot{q}[11,16]$. Mathematically,

$$
\dot{\sigma}=K \dot{q},
$$

where $K$ is a unitless proportional navigation constant.

The model of the motion relationship between the projectile and the target in three-dimensional space is shown in Figure 2. $P$ represents a moving projectile that is fired from a launcher towards a $\operatorname{target} T . V_{M}$ and $V_{T}$ represent the magnitudes of the projectile velocity and the target velocity vector, respectively, in the inertial reference frame $O x_{E} y_{E} z_{E}$. The components of the projectile position and the target position as defined in the inertial reference frame are $\left(x_{P}, y_{P}, z_{P}\right)^{T}$ and $\left(x_{T}, y_{T}, z_{T}\right)^{T}$, respectively. $\left(V_{P x}, V_{P y}, V_{P z}\right)^{T}$ and $\left(V_{T x}, V_{T y}, V_{T z}\right)$ represent the components of the projectile velocity vector $\mathbf{V}_{M}$ and the target velocity vector $\mathbf{V}_{T}$, respectively.

The LOS angular rate, $\dot{q}$, consists of the rates of change in the pitch angle $q_{\theta}$ and the yaw angle $q_{\psi} . q_{\theta}$ is the angle between the LOS and the horizontal plane. When $q_{\theta}$ is greater than zero, the LOS points above the horizontal plane. $q_{\psi}$ is the angle between the projections of the LOS onto the horizontal and vertical planes. If $q_{\psi}$ is greater than zero, then the LOS points to the right of the vertical plane when viewed from the rear. $r$ is the magnitude of the LOS.

To describe the geometric relationship between the projectile velocity vector and the LOS, the right-handed rectangular coordinate system $O x_{V} y_{V} z_{V}$ with its origin located at the center of mass is introduced, as shown in Figure 2. The $x$-axis, $O x_{V}$, is directed along the projectile's velocity vector. The $z$-axis, $O z_{V}$, which always remains in the vertical plane, is perpendicular to the $x$-axis and points downward. The $y$-axis is perpendicular to the $O x_{V}$ and $O z_{V}$ axes, pointing rightward. The sequence of rotations from the inertial reference frame consists of rotations through the ballistic yaw angle $\psi_{V}$ and the ballistic yaw angle $\theta_{V}$. The transformation matrix from $O x_{E} y_{E} z_{E}$ to $O x_{V} y_{V} z_{V}$ can be described as follows:

$$
T_{E}^{V}=\left[\begin{array}{ccc}
\cos \theta_{v} \cos \psi_{v} & \cos \theta_{v} \sin \psi_{v} & -\sin \theta_{v} \\
-\sin \psi_{v} & \cos \psi_{v} & 0 \\
\sin \theta_{v} \cos \psi_{v} & \sin \theta_{v} \sin \psi_{v} & \cos \theta_{v}
\end{array}\right] .
$$

3.1. PNG Law Formulation. For a fin-stabilized projectile with a low spin rate, the mutual coupling effects between the pitching and yawing motions are relatively weak and can be ignored $[2,13,16]$. Accordingly, the three-dimensional PNG problem can be decoupled into two two-dimensional problems in the vertical and horizontal planes of the velocity vector without loss of convergence $[2,6,17-20]$.
The position of the target with respect to the projectile in the inertial frame is defined as shown in the following equation:

$$
\mathbf{r}=\left[\begin{array}{l}
r_{x} \\
r_{y} \\
r_{z}
\end{array}\right]=\left[\begin{array}{c}
x_{P}-x_{T} \\
y_{P}-y_{T} \\
z_{P}-z_{T}
\end{array}\right] .
$$

The projectile's closing velocity vector $\mathbf{V}_{T M}$ is expressed as follows:

$$
\mathbf{V}_{T P}=\left[\begin{array}{c}
\dot{r}_{x} \\
\dot{r}_{y} \\
\dot{r}_{z}
\end{array}\right]=\left[\begin{array}{c}
V_{T P x} \\
V_{T P y} \\
V_{T P z}
\end{array}\right]=\left[\begin{array}{c}
V_{P x}-V_{T x} \\
V_{P y}-V_{T y} \\
V_{P z}-V_{T z}
\end{array}\right] .
$$

The time derivative of (12) provides the rate of change of $r$ as follows:

$$
\dot{r}=\frac{\left(r_{x} V_{T P x}+r_{y} V_{T P y}+r_{z} V_{T P z}\right)}{r} .
$$

As seen from the geometric relationship in Figure 2, the pitch angle $q_{\theta}$ and the yaw angle $q_{\psi}$ of the LOS are expressed as follows:

$$
\begin{aligned}
& q_{\theta}=\arctan \frac{r_{z}}{\sqrt{r_{x}^{2}+r_{y}^{2}}}, \\
& q_{\psi}=\arctan \frac{r_{y}}{r_{x}} .
\end{aligned}
$$

The time derivatives of (15) and (16) provide the rates of change of $q_{\theta}$ and $q_{\psi}$ as follows:

$$
\begin{aligned}
& \dot{q}_{\theta}=\frac{V_{T P y}\left(r_{x}^{2}+r_{y}^{2}\right)-r_{z}\left(r_{x} V_{T P x}+r_{y} V_{T P y}\right)}{r^{2} \sqrt{r_{x}^{2}+r_{y}^{2}}}, \\
& \dot{q}_{\psi}=\frac{r_{y} V_{T P x}-r_{x} V_{T P y}}{r_{x}^{2}+r_{y}^{2}} .
\end{aligned}
$$

Thus, the vertical- and horizontal-plane guidance laws can be expressed as shown in the following equations:

$$
\begin{gathered}
\dot{\theta}_{V}=K_{\theta} \dot{q}_{\theta}, \\
\dot{\psi}_{V}=K_{\psi} \dot{q}_{\psi},
\end{gathered}
$$

where $K_{\theta}$ and $K_{\psi}$ are the unitless proportional navigation constants for the vertical- and horizontal-plane guidance laws, respectively.

3.2. Gravity Compensation. Changes in the projectile and target velocities due to gravity cause the LOS to rotate. Considering the effect of gravity in the PNG law could allow unnecessary accelerations to be avoided in the correction process, thus reducing the requirements placed on the actuators [11]. Accordingly, a PNG law with gravity compensation is given below. 


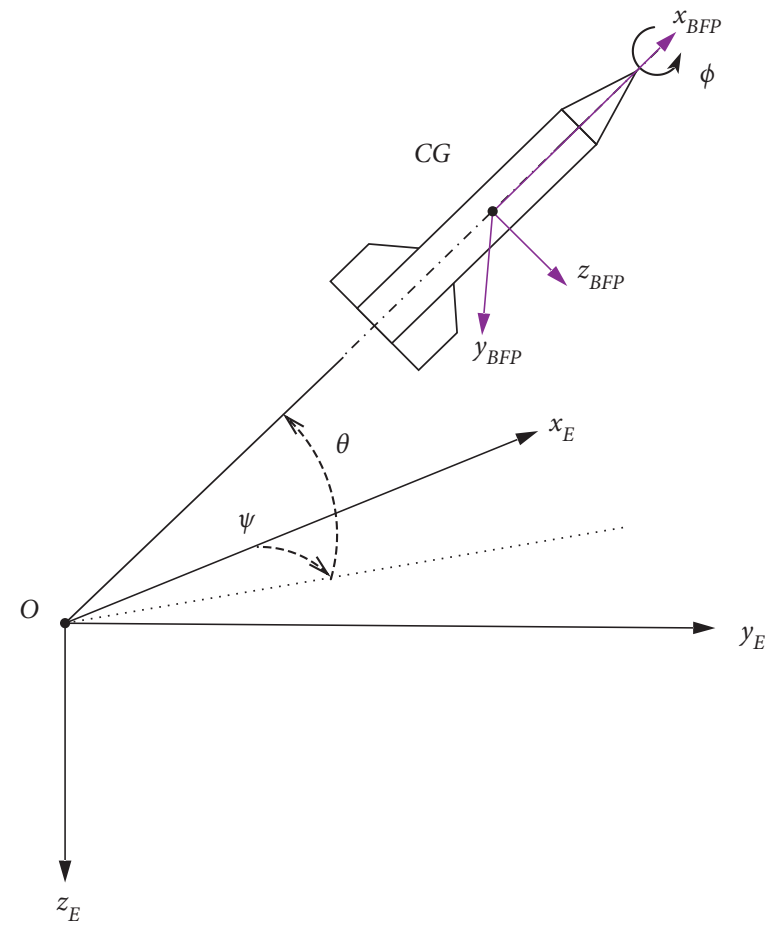

FIGURE 1: Schematic of the projectile's position coordinates.

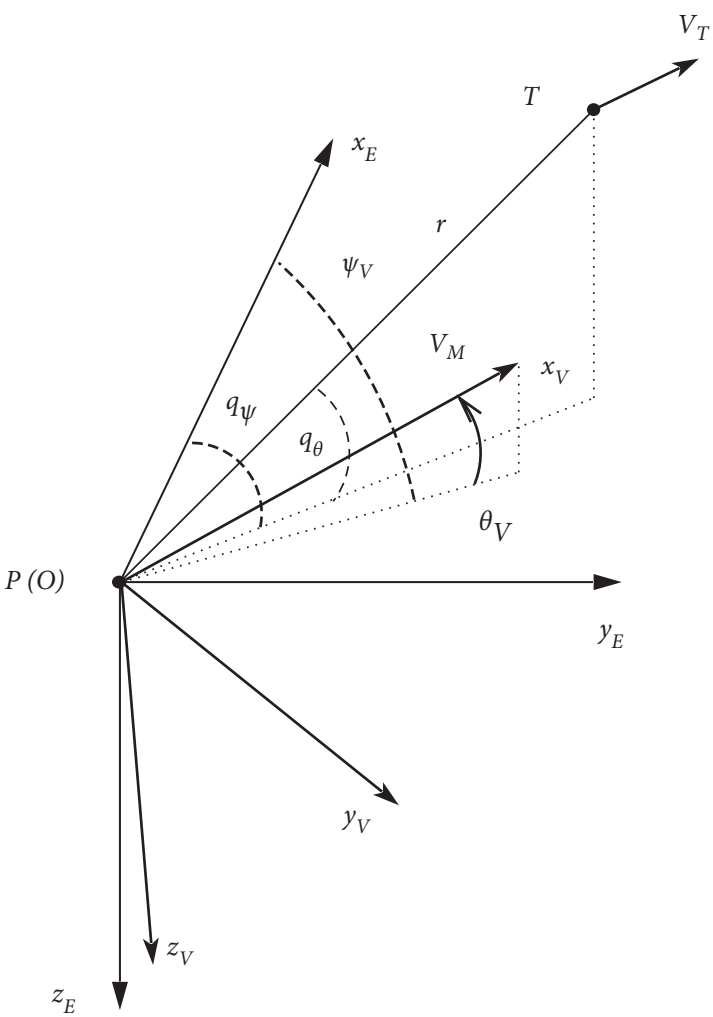

FIgURE 2: Schematic of the relative motion of the mortar projectile and the target. 
The components of the gravity acting on the projectile in $O x_{V} y_{V} z_{V}$ are expressed as follows:

$$
T_{E}^{V}=\left[\begin{array}{ccc}
\cos \theta_{v} \cos \psi_{v} & \cos \theta_{v} \sin \psi_{v} & -\sin \theta_{v} \\
-\sin \psi_{v} & \cos \psi_{v} & 0 \\
\sin \theta_{v} \cos \psi_{v} & \sin \theta_{v} \sin \psi_{v} & \cos \theta_{v}
\end{array}\right],
$$

where $\left(X_{G_{E}}, Y_{G_{E}}, Z_{G_{E}}\right)^{T}$ are the gravitational components in $O x_{E} y_{E} z_{E}$. It can be seen from (20) that gravity affects only the vertical-plane guidance law.

Because the effect of gravity on the relative motion between the projectile and the target is nonlinear, a nonlinear optimal guidance law design method is usually used to solve this problem. However, for trajectory correction projectiles, the capabilities of such methods are limited due to their small size and low onboard computing power. Thus, a simplified yet effective method is adopted in this article [16]. The optimal control variable $\dot{\theta}_{V}$ can be decomposed into control and gravity components, as shown in the following equation:

$$
\dot{\theta}_{V}=\dot{\theta}_{c}+\dot{\theta}_{g}
$$

where $\dot{\theta}_{V}$ is the rate of change in $\theta_{V}$ required by the optimal guidance law, $\dot{\theta}_{g}$ is the rate of change in $\theta_{V}$ due to gravity, and $\dot{\theta}_{c}$ is the rate of change in $\theta_{V}$ driven by the control system.

Compared to gravity, the lift force acting on the projectile is relatively small, so the effect of the lift on the rate of change in $\theta_{V}$ is negligible. Thus, $\dot{\theta}_{g}$ can be expressed as follows:

$$
\dot{\theta}_{g}=\frac{-g \cos \theta_{V}}{V} .
$$

By substituting (18) and (22) into (21) and simplifying, the vertical-plane PNG law with gravity compensation can be expressed as follows:

$$
\dot{\theta}_{c}=K_{\theta} \dot{q}_{\theta}+\frac{g}{V} \cos \theta_{V} .
$$

The guidance acceleration commands in the horizontal and vertical planes are determined by the following equations:

$$
\begin{aligned}
& n_{y c}=\frac{V}{g} \dot{\psi}_{V} \cos \theta_{V}=K_{\psi} \dot{q}_{\psi} \frac{V}{g} \cos \theta_{V}, \\
& n_{z c}=-\frac{V}{g} \dot{\theta}_{c}=-K_{\theta} \dot{q}_{\theta} \frac{V}{g}-\cos \theta_{V} .
\end{aligned}
$$

The off-axis acceleration command can then be decomposed into a magnitude and phase angle in accordance with the following equations:

$$
\begin{aligned}
\Gamma_{c} & =\sqrt{n_{y c}^{2}+n_{z c}^{2}}, \\
\phi_{N} & =\arctan \frac{n_{z c}}{n_{y c}}
\end{aligned}
$$

The process of applying the PNG law with gravity compensation is depicted in Figure 3. The parts outlined with dotted lines represent uplink data obtained before launch.

3.3. Optimization Schemes. For a class of dual-spin projectiles equipped with fixed canards, the control force provided by one pair of canards with a fixed cant angle can be oriented in any direction at any time and continuously adjusted. However, the limited control force cannot generate a sufficient commanded acceleration to successfully hit the target point when the above PNG law is used [4]. Because fixed canards cannot ensure the desired accelerations, $K_{\theta}$ and $K_{\psi}$ are different from the guidance constants of the classical PNG laws previously discussed. Whereas the conventional proportional navigation constants are chosen empirically, this approach is not valid for projectiles equipped with fixed canards.

In essence, the proper selection of the proportional navigation constants can be formulated as a nonlinear optimization problem. In this optimization problem, the proportional navigation constants are treated as the design variables $\mathbf{x}$, defined as follows:

$$
\mathbf{x}=\left(K_{\theta}, K_{\psi}\right)^{T} .
$$

The basic task is to seek the set of proportional navigation constants that minimizes the objective function, which represents a difference metric. To solve this optimization problem, two optimization schemes are introduced for the construction of corresponding optimization models.

The target is assumed to be stationary, namely, the velocity and acceleration of the target are assumed to be equal to zero. In the optimization design process for the PNG law, the nominal trajectory is the uncontrolled trajectory subject to no initial value perturbations. The impact point of the nominal trajectory is regarded as the target point $\mathbf{T}$, and the component form is $\mathbf{T}=\left(x_{T}, y_{T}, z_{T}\right)$.

3.3.1. Scheme 1. In scheme 1, the objective function is the sum squared error defined in the following equation:

$$
J_{1}(\mathbf{x})=\frac{1}{2}[\mathbf{P}-\mathbf{T}]^{T}[\mathbf{P}-\mathbf{T}]
$$

where $\mathbf{P}$ is the impact point of the trajectory with initial value perturbations under control (in component form, $\left.\mathbf{P}=\left(x_{P}, y_{P}, z_{P}\right)\right)$ and $\mathbf{P}-\mathbf{T}$ is the distance between $\mathbf{P}$ and $\mathbf{T}$. To find a representative perturbed trajectory, initial value perturbations that cover the maximum dispersion deviation can be selected. Note that it is assumed that the proportional navigation constants for the perturbed trajectory are also applicable to other trajectories.

For scheme 1, the essence of the optimization problem is to find a set of optimal PNG law constants that minimize the miss distance. The corresponding optimization model can be expressed as follows: 


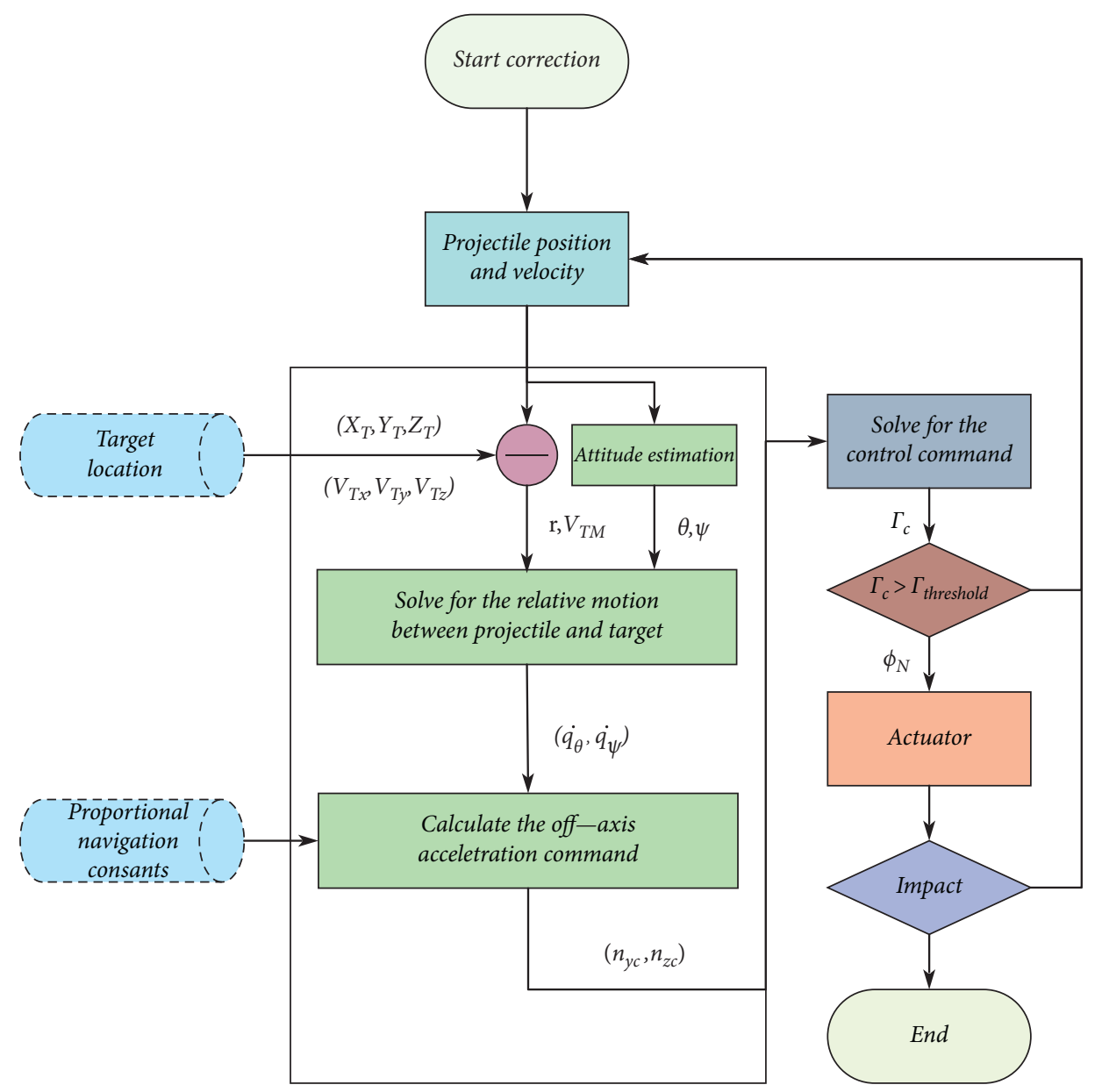

FIGURE 3: The process of applying the PNG law with gravity compensation.

$$
\begin{cases}\min & J_{1}(\mathbf{x}, \mathbf{P})=\min \frac{1}{2}[\mathbf{P}-\mathbf{T}]^{T}[\mathbf{P}-\mathbf{T}] \\
\text { subject to }\left\{\begin{array}{l}
K_{\theta} \in[-5,5], \\
K_{\psi} \in[-5,5] .
\end{array}\right.\end{cases}
$$

A suitable constraint range of the design variables is necessary to comprehensively evaluate the entire parameter spaces. According to previous scientific research and engineering articles, the range $[-5,5]$ is sufficient to satisfy the present case $[11,16]$.

3.3.2. Scheme 2. The most important performance specification is the miss distance, which is defined by the weapon system requirements. The miss distance for a sample of projectile firings is typically measured as the CEP [21]. Therefore, the CEP is adopted as the objective function in optimization scheme 2 , as shown in the following equation:

$$
J_{2}(\mathbf{x}, \mathbf{P})=\operatorname{CEP}(\mathbf{x}, \mathbf{P}),
$$

where CEP is assumed to be a nonlinear function of the PNG law constants, $\mathbf{x}$, and the impact point under control, $\mathbf{P}$.

For scheme 2, the essence of the optimization problem is to find a set of optimal PNG law constants that minimize the CEP. The CEP metric is determined through Monte Carlo analysis. The corresponding optimization model can be expressed in the following form:

$$
\begin{cases}\min & J_{2}(\mathbf{P})=\min \operatorname{CEP}(\mathbf{P}) \\
\text { subject to } & \left\{\begin{array}{l}
K_{\theta} \in[-5,5], \\
K_{\psi} \in[-5,5] .
\end{array}\right.\end{cases}
$$

The configurations of the two proposed optimization schemes are presented in Table 1.

At this step, an optimization algorithm is required to solve the optimality equations (30) and (32). Since these equations are nonlinear, intelligent optimization algorithms, such as NSGA-II $[22,23]$ and the differential evolution (DE) [24-26], and particle swarm optimization (PSO) algorithms [27] are relatively better choices than traditional methods. For the present single objective problem, the DE algorithm might be easier to deploy. The $\mathrm{DE}$ algorithm is a populationbased intelligent optimization algorithm designed by Storn 
TABle 1: Configurations of the two proposed optimization schemes.

\begin{tabular}{lccc}
\hline Scheme & Objective function & $\begin{array}{c}\text { Design } \\
\text { variables }\end{array}$ & Constraint \\
\hline Scheme 1 & $\min (1 / 2)[\mathbf{P}-\mathbf{T}]^{T}[\mathbf{P}-\mathbf{T}]$ & $K_{\theta}, K_{\psi}$ & {$[-5,5]$} \\
Scheme 2 & $\operatorname{min~CEP~}(\mathbf{P})$ & $K_{\theta}, K_{\psi}$ & {$[-5,5]$} \\
\hline
\end{tabular}

and Price as a simple and effective evolutionary algorithm for real-valued optimization problems. The DE algorithm can search for the globally optimal solution while avoiding the complex computation of auxiliary gradient information. Additionally, DE requires few control parameters and converges rapidly. In this paper, the DE algorithm is used to solve the optimality equations. The flowchart of the optimization task based on the DE algorithm is shown in Figure 4.

In the $\mathrm{DE}$ algorithm, the random method is used to initialize the population and perform the mutation and crossover operations. For usual cases, the design of experiments (DOE) method has a wider application range; furthermore, as shown in $[28,29]$, the DOE method can improve the optimization efficiency. For the present case, there are only two design variables $\left(K_{\theta}\right.$ and $\left.K_{\psi}\right)$. Meanwhile, since the guided flight dynamic model is relatively simple and the time required for solving this model one time is less than approximately $1 \mathrm{sec}$, the random method in the DE algorithm instead of the DOE method is directly used to reduce the design space.

\section{Results and Discussion}

The projectile used in this study is a $120 \mathrm{~mm}$, fin-stabilized mortar projectile The physical properties and aerodynamic coefficients of this projectile are listed in Tables 2 and 3, respectively.

The unguided mortar projectile, on its nominal trajectory, is launched at a quadrant elevation of approximately $45^{\circ}$ and a muzzle velocity of $341.0 \mathrm{~m} / \mathrm{s}$. The initial conditions for the nominal trajectory are shown in Table 4 . The corresponding impact point is at $(7294.1,0,0) \mathrm{m}$. As a measure of performance, the CEP of an unguided round is approximately $58.2 \mathrm{~m}$, as shown in Figure 5 . For scheme 1, the selected initial value perturbations are shown in Table 4.

For scheme 2, Monte Carlo simulations are conducted using the nonlinear 6-DOF model of the projectile. Each Monte Carlo run consists of flying the shell 300 times with particular standard deviations of the perturbations under study. For each perturbed parameter, the error is modeled as a random number generated using a standard normal distribution. The standard deviations applied in this analysis are listed in Table 5.

In addition, both GPS and geomagnetic noises are considered in the stochastic Monte Carlo simulations. The GPS model and the geomagnetic model both include random error terms for position and velocity and for roll angle, respectively. The measurement noise is modeled using zeromean Gaussian distributions with the standard deviations listed in Table 6. The position and velocity of the projectile are provided by a GPS receiver in flight at a rate of $10 \mathrm{~Hz}$. The roll angle is provided by a geomagnetic sensor at a rate of $200 \mathrm{~Hz}$. A $10 \mathrm{~Hz}$ update rate is used for the guidance and control algorithm.

The DE algorithm with a parallel computational architecture is used to solve the optimization problem for the PNG law constants. Detailed information about the parameters and the basic flow can be found in [24-26]. Table 7 specifies the initialization of the parameters implemented in the DE algorithm.

The convergence characteristics achieved with the two schemes based on the DE algorithm can be visualized in Figure 6 and are listed in Table 8. Both schemes converge efficiently and have good convergence characteristics. For scheme 1, the optimal objective function value is approximately 35.3 , and $K_{\theta}$ and $K_{\psi}$ are 2.69 and -1.61 , respectively. This means that the radial distance between the guided impact point under the optimal proportional navigation constants from scheme 1 , and the target is approximately $35.3 \mathrm{~m}$. It is possible that the deviation is so large that the current correction capabilities are not sufficient. For scheme 2 , the optimal objective function value is approximately 2.94 , and $K_{\theta}$ and $K_{\psi}$ are 2.45 and -3.88 , respectively.

To demonstrate the guidance performance, the unguided and guided trajectories for the example mortar configuration specified in Table 5 are presented for comparison in Figure 7, and the specific data are listed in Table 7. The figure shows the nominal trajectory, the unguided trajectory, and the PNG-guided trajectories using schemes 1 and 2. The nominal trajectory was simulated using the mean values shown in Table 5. The unguided and guided trajectories were simulated using the perturbed values shown in Table 5 .

Figures $7(\mathrm{a})$ and $7(\mathrm{~b})$ plot the mortar altitude and crossrange versus downrange. The miss distances for the unguided, scheme 1, and scheme 2 trajectories are $44.3 \mathrm{~m}$, $4.7 \mathrm{~m}$, and $1.5 \mathrm{~m}$, respectively. Compared to the unguided trajectory, the guided mortar trajectories under both schemes closely approach the target, with impact errors on the order of a few meters. Note that scheme 2 shows better range correction performance. Comparisons of pitch and yaw attitude for the unguided and guided trajectories are provided in Figures 7(c) and 7(d). While the nominal and unguided trajectories show a steady decrease in pitch and yaw attitude after the trajectory apex, the guided trajectories show oscillatory responses due to the continuous correction of the fixed canards. The impact angles for schemes 1 and 2 are $-49.6^{\circ}$ and $-53.2^{\circ}$, respectively. The aerodynamic angles of attack are shown in Figure 7(e). The angles of attack for the nominal and unguided cases remain relatively small and below $0.5^{\circ}$. For both guidance schemes, the oscillatory amplitudes are similar and below $2^{\circ}$, while the oscillatory frequency for scheme 1 is somewhat lower.

To compare the performance of the two schemes, Monte Carlo simulations were conducted with the uncertainties in the initial conditions shown in Table 5 and the measurement noise characteristics shown in Table 6 . Table 9 and Figure 5 present the impact points for the unguided and guided mortar configurations with a sample size of 300 . The CEP is defined as the radius of a circle 


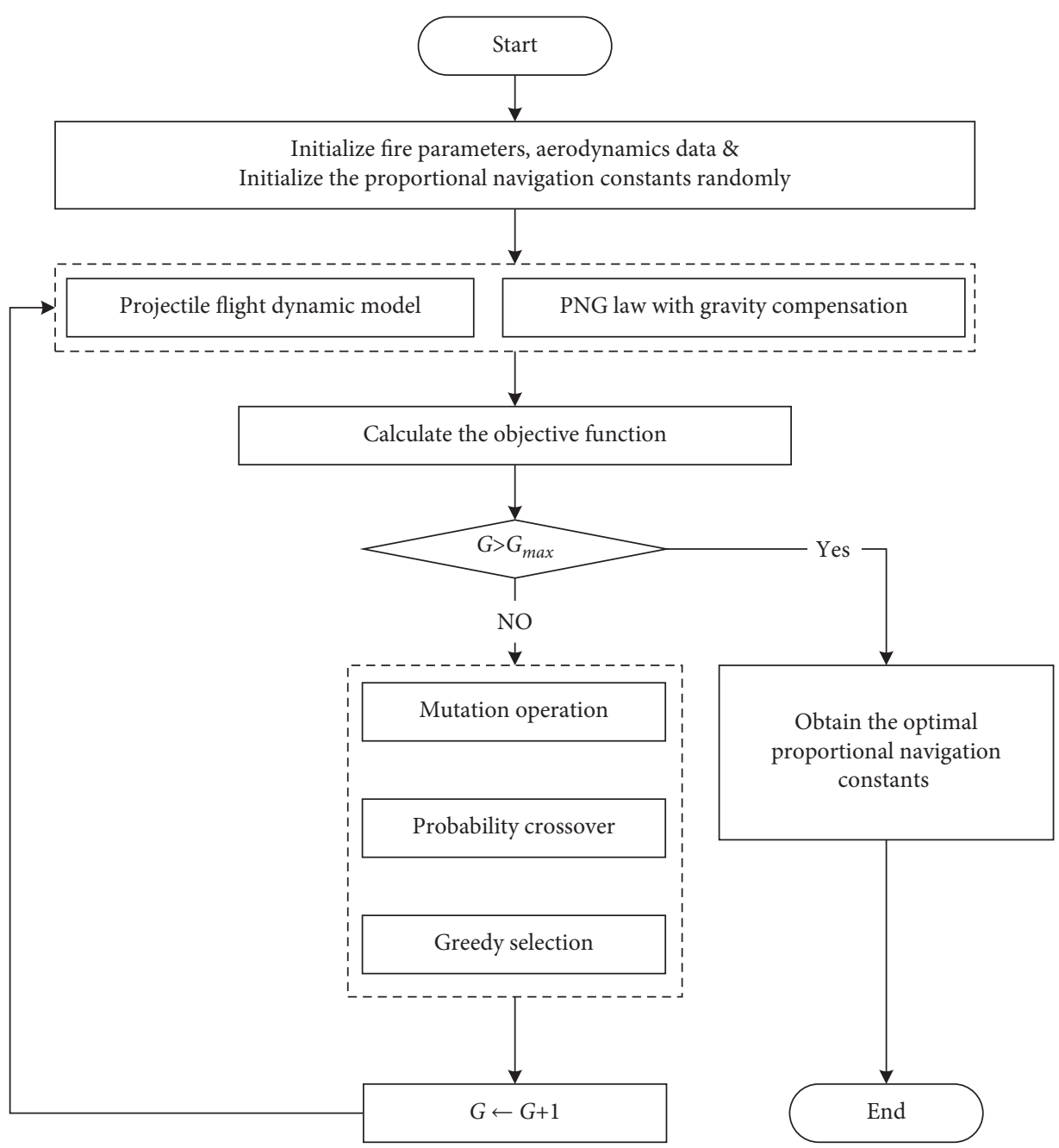

FIgURE 4: The process of applying the PNG law with gravity compensation.

Table 2: Physical properties of the projectile.

\begin{tabular}{lcccc}
\hline$m(\mathrm{~kg})$ & $I_{A}\left(\mathrm{~kg} \bullet \mathrm{m}^{2}\right)$ & $I_{C}\left(\mathrm{~kg} \bullet \mathrm{m}^{2}\right)$ & CG from nose $(\mathrm{m})$ & $D(\mathrm{~m})$ \\
\hline 14.72 & 0.022 & 0.2 & 0.295 & 0.120 \\
\hline
\end{tabular}

TABle 3: Aerodynamic coefficients of the $120 \mathrm{~mm}$ mortar projectile.

\begin{tabular}{lcccccc}
\hline Mach & 0.5 & 0.6 & 0.7 & 0.8 & 0.9 & 0.0 \\
\hline$C_{D}$ & 0.0929 & 0.0904 & 0.092 & 0.1707 & 0.3193 & 2.9442 \\
$C_{l \alpha}$ & 2.9153 & 2.9492 & 2.9696 & 2.9752 & -0.4849 \\
$C_{y p a}$ & -0.0166 & -0.0166 & -0.0150 & -0.01354 & -5.572 & -5.998 \\
$C_{m \alpha}$ & -5.056 & --5.178 & -5.266 & -0.040 & -0.044 & -6.00907 \\
$C_{n p \alpha}$ & -0.040 & -0.040 & -0.040 & 102.645 & -0.649 \\
$C_{m q}$ & 92.706 & 95.565 & 97.591 & $-2.0 \mathrm{E}-4$ & $-2.1 \mathrm{E}-4$ & 0.051 \\
$C_{l D D}$ & $-2.1 \mathrm{E}-4$ & $-2.1 \mathrm{E}-4$ & $-2.1 \mathrm{E}-4$ & 0.004 & 0.004 & $-2.1 \mathrm{E}-4$ \\
$C_{C C A}$ & 0.005 & 0.005 & 0.004 & 0.195 & 0.208 \\
$C_{C C N \delta}$ & 0.230 & 0.200 & 0.200 & 0.23226 & 0.23247 \\
$x_{C c p}$ & 0.23163 & 0.23184 & 0.23205 & & 0.221 \\
\hline
\end{tabular}


TABLE 4: Initial conditions for scheme 1.

\begin{tabular}{lcc}
\hline State & Nominal & Perturbated \\
\hline Launch elevation angle (degrees) & 45.0 & 46.0 \\
Launch azimuth angle (degrees) & 0.0 & 0.0 \\
Muzzle velocity (m/s) & 341.0 & 338.0 \\
Wind & 0.0 & 9.0 \\
Axial force coefficient multiplier & 1.0 & 1.1 \\
\hline
\end{tabular}

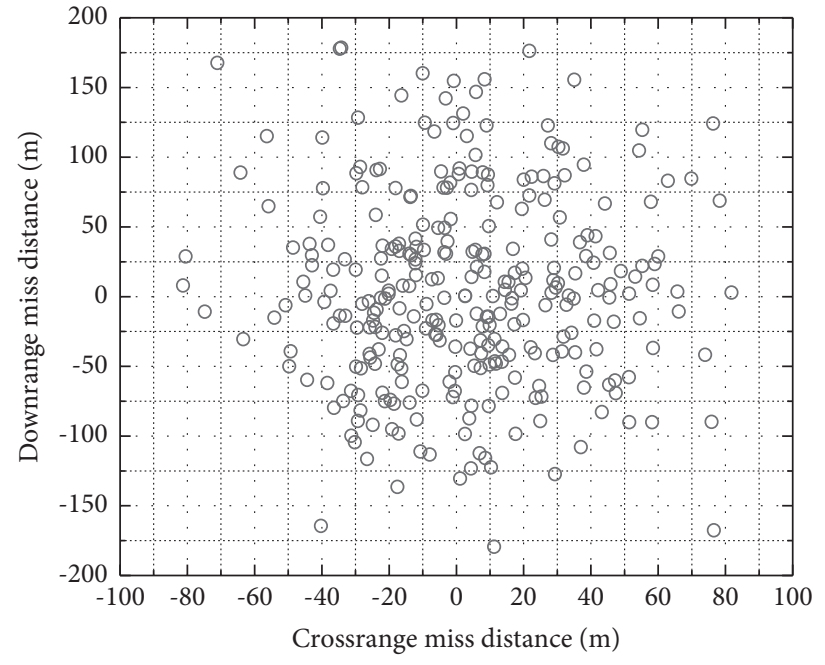

$\circ$ Unguided

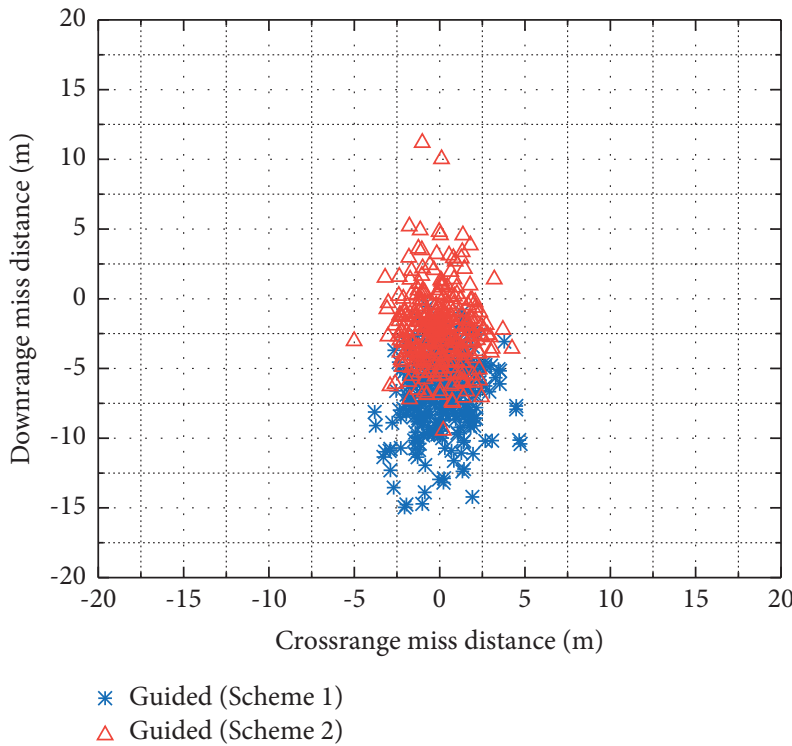

(b)

FIGURE 5: Impact point dispersion for unguided flights and guided flights under both schemes: (a) unguided flights and (b) guided flights for schemes 1 and 2 .

Table 5: Perturbation standard deviations.

\begin{tabular}{lcc}
\hline State & Nominal & Perturbated \\
\hline Muzzle velocity (m/s) & 341.0 & 1.00 \\
Launch elevation angle (degrees) & 45.0 & 0.30 \\
Launch azimuth angle (degrees) & 0.0 & 0.30 \\
Launch elevation angle rate (degrees/s) & 0.0 & 0.33 \\
Launch azimuth angle rate (degrees/s) & 0.0 \\
Mass & 14.72 \\
Axial & \\
Normal & \\
Pitch & - \\
Axial for canards & \\
Normal for canards & \\
Pitch for canards & \\
Wind (m/s) & 0.33 \\
Diameter (m) & 0.120 \\
\hline
\end{tabular}

TABLE 6: Standard deviations for GPS and geomagnetic measurements.

\begin{tabular}{lr}
\hline Measurement signal & Standard deviation \\
\hline$x(\mathrm{~m})$ & 2.24 \\
$y(\mathrm{~m})$ & 2.24 \\
$z(\mathrm{~m})$ & 2.83 \\
$v_{x}(\mathrm{~m} / \mathrm{s})$ & 0.32 \\
$v_{y}(\mathrm{~m} / \mathrm{s})$ & 0.32 \\
$v_{z}(\mathrm{~m} / \mathrm{s})$ & 0.32 \\
$\phi$ (degrees) & 1.67 \\
\hline
\end{tabular}


TABLE 7: Initialization of the parameters for the DE algorithm.

\begin{tabular}{lc}
\hline Parameter & Scheme $1 / 2$ \\
\hline Number of individuals (NP) & 30 \\
Weight factor $(F)$ & 0.5 \\
Crossover rate $(\mathrm{CR})$ & 0.9 \\
Mutation strategy & DE/rand/1/exp \\
Selection strategy & Best-parent-child \\
Maximum number of generations $\left(G_{\max }\right)$ & 100 \\
Termination criteria & $G>G_{\text {max }} \| J(x)<1 E-6$ \\
\hline
\end{tabular}

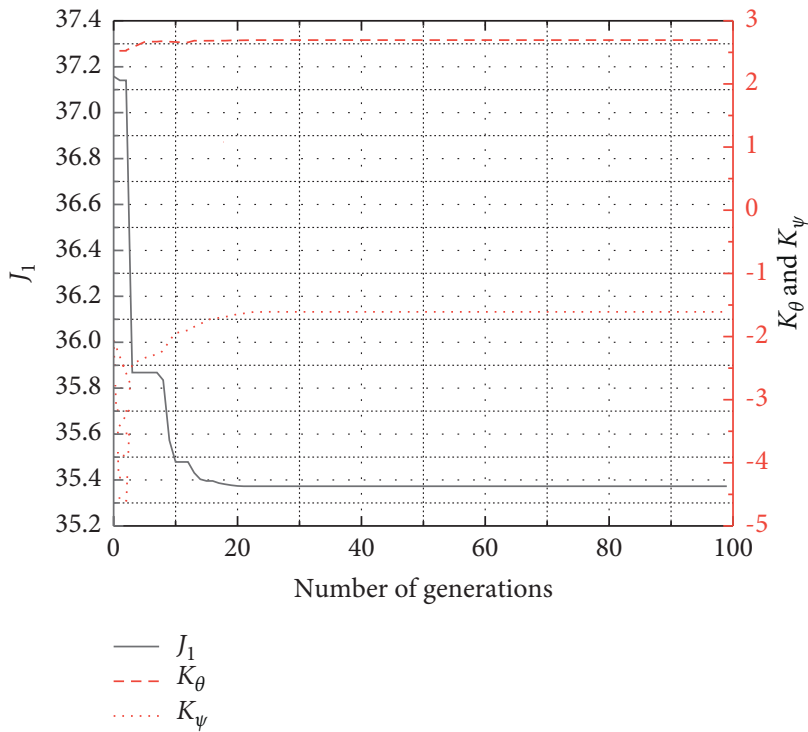

(a)

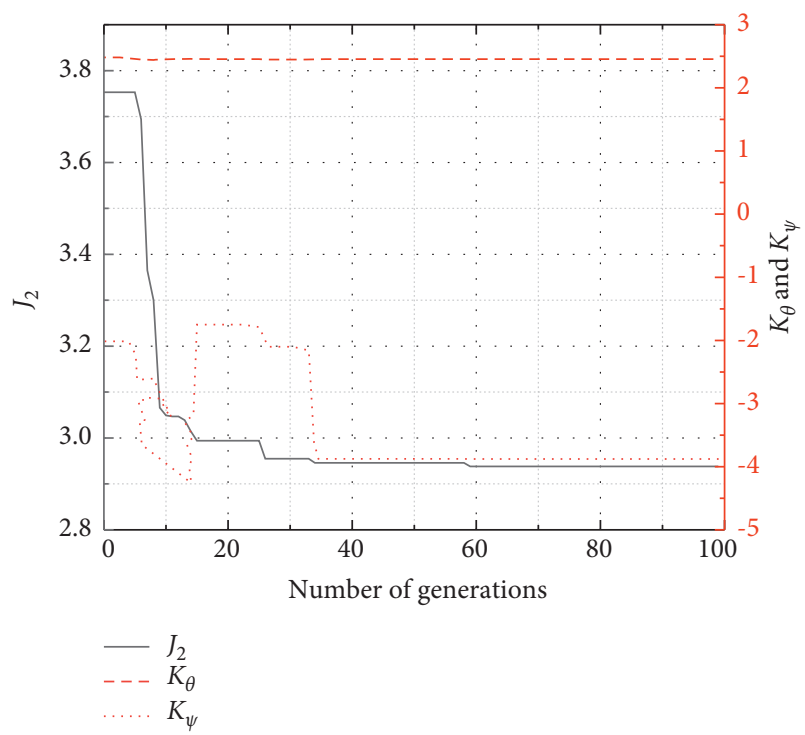

(b)

Figure 6: Convergence characteristics for schemes 1 and 2 with the DE algorithm: (a) scheme 1 and (b) scheme 2.

TABLE 8: Convergence characteristics and ballistic performance comparison between schemes 1 and 2 .

\begin{tabular}{lccc}
\hline Parameter/objective & Unit & Scheme 1 & Scheme 2 \\
\hline Objective function value & $\mathrm{m}$ & 35.3 & 2.94 \\
$K_{\theta}$ & 1 & 2.69 & 2.45 \\
$K_{\psi}$ & 1 & -1.61 & -3.88 \\
Miss distances & $\mathrm{m}$ & 4.7 & 1.5 \\
Impact angle & degrees & -49.6 & -53.2 \\
\hline
\end{tabular}

centered on the mean impact point that contains $50 \%$ of the impact points [30]. The CEP for the unguided case is approximately $58.2 \mathrm{~m}$. For both schemes, the CEP is no greater than $10 \mathrm{~m}$. The CEP in the guided case under scheme 1 is reduced to $6.9 \mathrm{~m}$, while the CEP in the guided case under scheme 2 is reduced to $2.9 \mathrm{~m}$. The reduction in the CEP for both schemes indicates their effectiveness. Note that the guided case under scheme 2 shows a greater reduction in the CEP.
In Figure 8, the dispersion is presented in the form of histograms. The upper and lower panels of Figure 8 represent the unguided case and the guided cases under schemes 1 and 2, respectively. It can be seen that while the dispersions in the guided cases are relatively low, there are some outliers. The mean impact points in the guided cases under schemes 1 and 2 are $(6.7,0.14) \mathrm{m}$ and $(2.1,0.01) \mathrm{m}$, respectively. The mean impact point under scheme 2 is closer to the impact point in the nominal case, as is also 

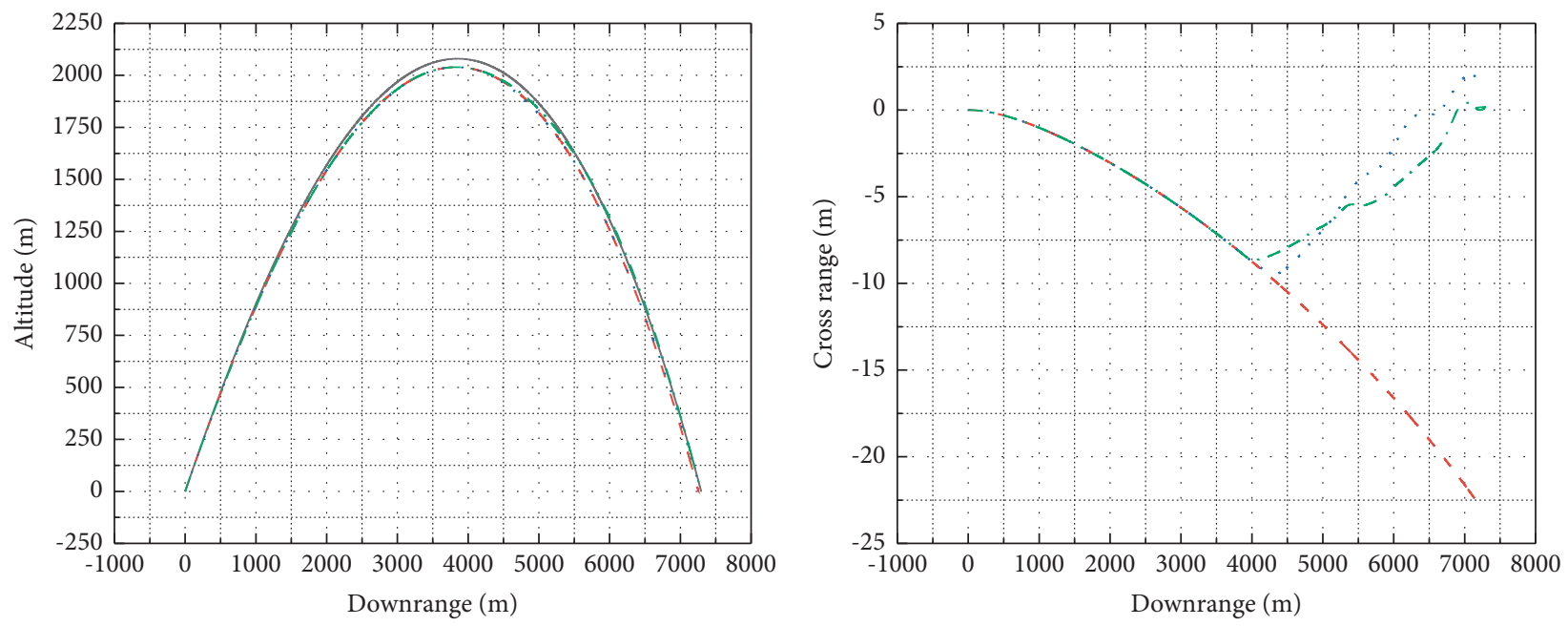

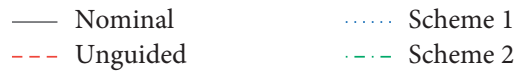

(a)

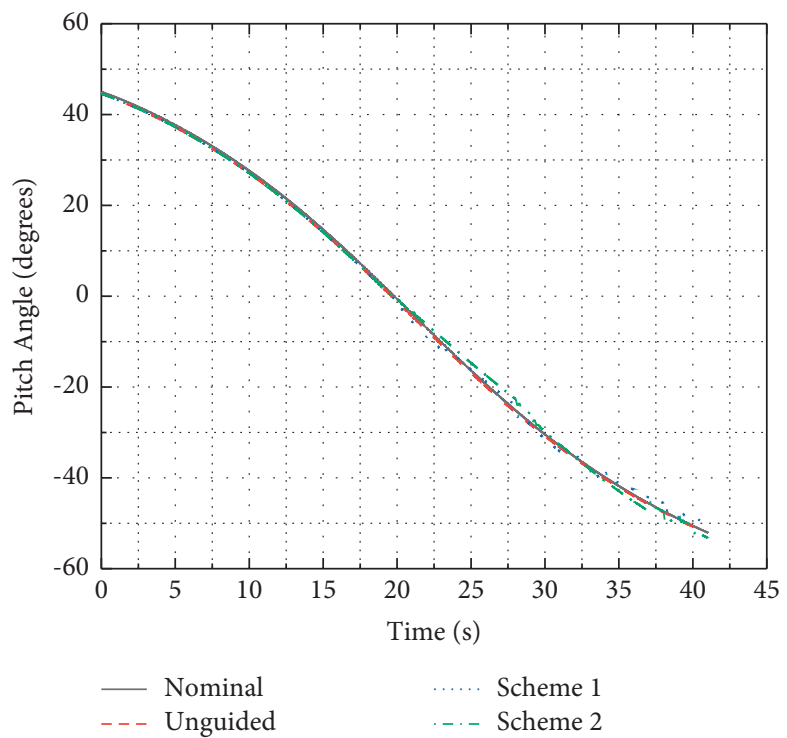

(c)

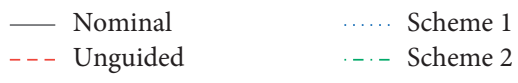

(b)

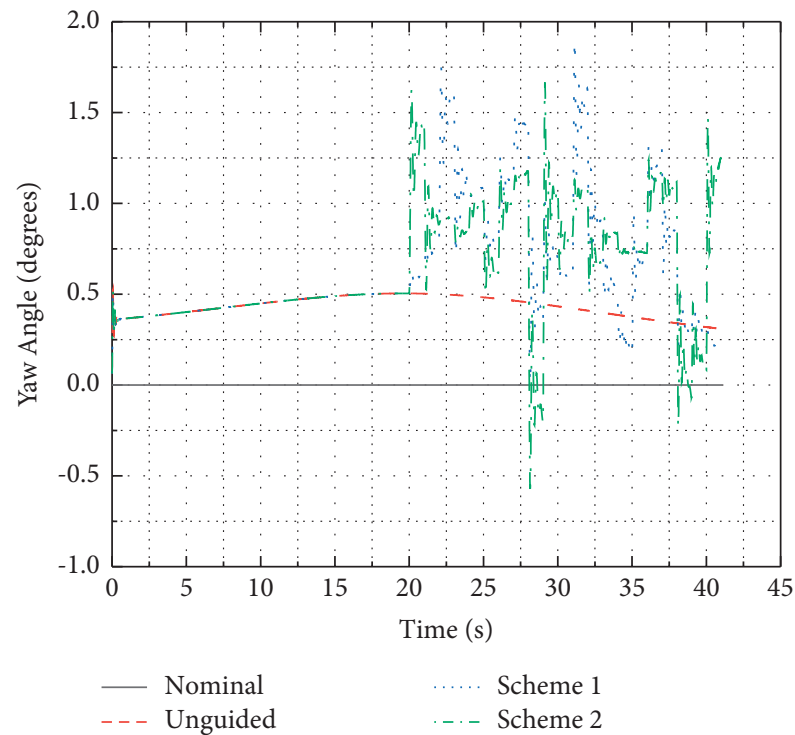

(d)

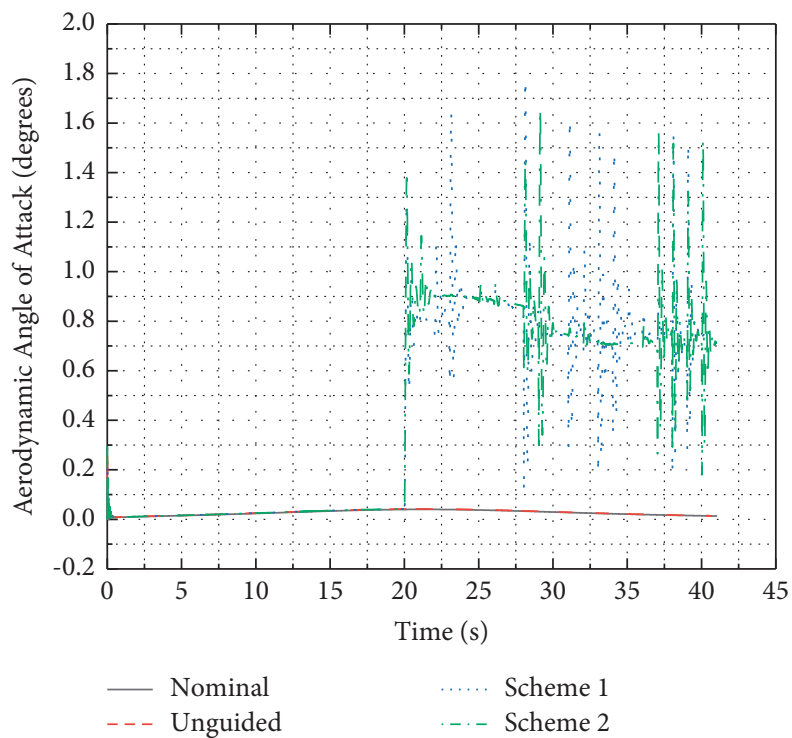

(e)

FIGURE 7: The ballistic performance for the unguided and guided trajectories: (a) altitude versus downrange, (b) crossrange versus downrange, (c) Euler pitch angle versus time, (d) Euler yaw angle versus time, and (e) aerodynamic angle of attack versus time. 
TABLE 9: Impact point dispersion comparison between schemes 1 and 2.

\begin{tabular}{lcccc}
\hline Parameter/Objective & Unit & Unguided & Scheme 1 & Scheme 2 \\
\hline Downrange error & $\mathrm{m}$ & 0.0 & 6.7 & 2.1 \\
Crossrange error & $\mathrm{m}$ & 0.0 & 0.14 & 0.01 \\
CEP & $\mathrm{m}$ & 58.2 & 6.9 & 2.9 \\
\hline
\end{tabular}

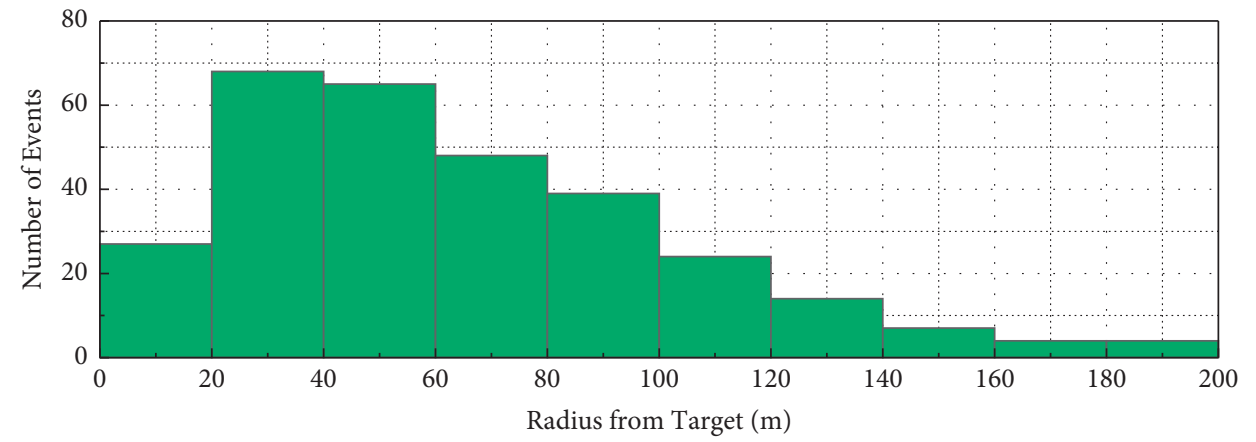

Unguided

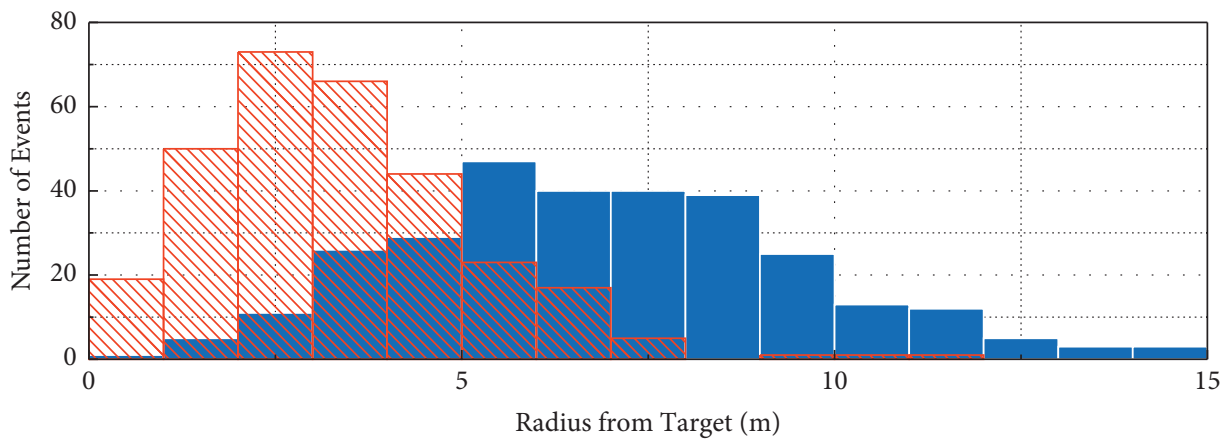

Guided (Scheme 1)

Guided (Scheme 2)

FIGURE 8: Histograms for unguided flights and guided flights under both schemes.

apparent from the impact point dispersion results shown in Figure 5.

\section{Conclusions}

In this paper, the selection of proportional navigation constants is formulated as an optimization problem instead of selecting these constants empirically. Based on a PNG law with gravity compensation, two optimization schemes are proposed in which the proportional navigation constants are taken as the design variables. The objective functions of schemes 1 and 2 are the sum squared error between the impact point and target and the CEP, respectively. Several guided trajectory simulations have been conducted with the optimized proportional navigation constants to investigate the performance of the two schemes. The results show that although the trajectory can be successfully guided to the target under both schemes, scheme 2 results in better range correction performance and less oscillation in the angle of attack. In addition, Monte Carlo simulations with uncertainties in the initial launch conditions, aerodynamics, environment, and measurements have been conducted for both schemes. The results show that the CEP under scheme 2 is approximately 2.4 times smaller than that under scheme 1 , and the mean impact point under scheme 2 is closer to the target. For the application of PNG optimization, it is suggested that future work should focus on the following objectives. First, the impact angle and angle of attack constraints should be considered in the objective function for the optimization of the proportional navigation constants. Second, the DOE method instead of the random method from the original DE algorithm should be investigated to reduce the design space. Third, a topological analysis of the objective function should be performed to improve the adaptability of scheme 1 .

\section{Data Availability}

The data used to support the findings of this study are available from the corresponding author upon request.

\section{Conflicts of Interest}

The authors declare that there are no conflicts of interest regarding the publication of this paper. 


\section{References}

[1] C. S. Foreman, David, and T. Yuri, "Trajectory-shaping and precision guidance of a spinning mortar without angle-state feedback," in Proceedings of the AIAA Guidance, Navigation, and Control Conference, Monterey, CA, USA, August 2011.

[2] Q.-W. Guo, W.-D. Song, Y. Wang, and Z.-C. Lu, "Guidance law design for a class of dual-spin mortars," International Journal of Aerospace Engineering, vol. 2015, pp. 1-12, Article ID 952076, 2015.

[3] Y. Zhang, M. Gao, S. Yang, and D. Fang, "An adaptive proportional navigation guidance law for guided mortar projectiles," The Journal of Defense Modeling and Simulation: Applications, Methodology, Technology, vol. 13, no. 4, pp. 467-475, 2016.

[4] F. Fresconi, "Guidance and control of a fin-stabilized projectile based on flight dynamics with reduced sensor and actuator requirements," in Proceedings of the AIAA Guidance, Navigation, and Control Conference, Dever, CO, USA, August 2012.

[5] R. Li, D. Li, and J. Fan, "Correction strategy of mortars with trajectory correction fuze based on image sensor," Sensors, vol. 19, no. 5, p. 1211, 2019.

[6] F. Fresconi, I. Celmins, S. Silton, and M. Costello, "High maneuverability projectile flight using low cost components," Aerospace Science and Technology, vol. 41, pp. 175-188, 2015.

[7] J. Rogers and M. Costello, "Design of a roll-stabilized mortar projectile with reciprocating canards," Journal of Guidance, Control, and Dynamics, vol. 33, no. 4, pp. 1026-1034, 2010.

[8] W. Park, C.-K. Ryoo, Y. Kim, B. Kim, and J. Kim, A New Practical Guidance Law For A Guided ProjectileAmerican Institute of Aeronautics and Astronautics, Reston, VA, USA, 2011.

[9] C. Jisi, S. Qiang, and Z. Pian, "Simulation for two-dimensional trajectory correction projectile with fixed-canard based on modified proportional navigation," in Proceedings of the 2017 IEEE International Conference on Unmanned Systems (ICUS), pp. 72-77, Beijing, China, October 2017.

[10] Y. Zhang, M. Gao, S. Yang, B. Li, and X. Gao, "A practical adaptive proportional-derivative guidance law," IEEE/CAA Journal of Automatica Sinica, vol. 5, p. 6, 2016.

[11] P. Zarchan, Tactical and Strategic Missile Guidance, American Institute of Aeronautics and Astronautics, Inc., Washington, DC, USA, Sixth edition, 2012.

[12] P. Wernert, S. Theodoulis, and Y. Morel, Flight Dynamics Properties of $155 \mathrm{Mm}$ Spin-Stabilized Projectiles Analyzed In Different Body FramesAmerican Institute of Aeronautics and Astronautics, Toronto, OT, Canada, 2010.

[13] R. L. McCoy, Modern Exterior Ballistics: The Launch and Flight Dynamics of Symmetric Projectiles, Schiffer Pub, Atglen, PA, USA, 2nd ed edition, 2012.

[14] P. Wernert, "Stability analysis for canard guided dual-spin stabilized projectiles," in AIAA Atmospheric Flight Mechanics ConferenceAmerican Institute of Aeronautics and Astronautics, Chicago, IL, USA, 2009.

[15] S. Theodoulis, F. Sève, and P. Wernert, "Robust gainscheduled autopilot design for spin-stabilized projectiles with a course-correction fuze," Aerospace Science and Technology, vol. 42, pp. 477-489, 2015.

[16] H. Zipeng, Exterior Ballistics of Projectile and Rockets, Beijing Institute of Technology Press, Beijing, China, 2008.

[17] X. Hu, S. Yang, F. Xiong, and G. Zhang, "Stability of spinning missile with homing proportional guidance law," Aerospace Science and Technology, vol. 71, pp. 546-555, 2017.
[18] D. V. Ćuk, "Trajectory correction of gyroscopic stabilized projectile using proportional navigation," Military Technical Courier, vol. 58, 2010.

[19] T. Jitpraphai, B. Burchett, and M. Costello, "A comparison of different guidance schemes for a direct fire rocket with a pulse jet control mechanism," in AIAA Atmospheric Flight Mechanics Conference and ExhibitAmerican Institute of Aeronautics and Astronautics, Montreal, Canada, 2001.

[20] F. Shengnan, L. Xiaodong, Z. Wenjie, and X. Qunli, "Multiconstraint adaptive three-dimensional guidance law using convex optimization," Journal of Systems Engineering and Electronics, vol. 31, no. 4, pp. 791-803, 2020.

[21] B. T. Burchett and M. F. Costello, Robust Lateral Pulse Jet Control of an Atmospheric Rocket, Doctor of Philosophy, Oregon State University, Corvallis, OR, USA, 2001.

[22] X. Sun, Z. Shi, G. Lei, Y. Guo, and J. Zhu, "Multi-objective design optimization of an IPMSM based on multilevel strategy," IEEE Transactions on Industrial Electronics, vol. 68, no. 1, pp. 139-148, 2021.

[23] K. Diao, X. Sun, G. Lei, Y. Guo, and J. Zhu, "Multiobjective system level optimization method for switched reluctance motor drive systems using finite-element model," IEEE Transactions on Industrial Electronics, vol. 67, no. 12, pp. 10055-10064, 2020.

[24] R. Storn and K. Price, "Differential evolution-a simple and efficient heuristic for global optimization over continuous spaces," Journal of Global Optimization, vol. 11, no. 4, pp. 341-359, 1997.

[25] R. Storn, K. Price, Differential Evolution Homepage, Differential Evolution Homepage. (2018). http://www.ICSI. Berkeley.edu/ storn/code.html.

[26] U. K. Chakraborty, Advances in Differential Evolution, Springer-Verlag, Berlin, Germany, 2008.

[27] R. Eberhart and J. Kennedy, "Particle swarm optimization," in Proceedings of the IEEE International Conference on Neural Networks, pp. 1942-1948, Shenzhen, China, June 1995.

[28] K. Diao, X. Sun, G. Lei, G. Bramerdorfer, Y. Guo, and J. Zhu, "Robust design optimization of switched reluctance motor drive systems based on system-level sequential taguchi method," IEEE Transactions on Energy Conversion, vol. 1, 2021.

[29] K. Diao, X. Sun, G. Lei, G. Bramerdorfer, Y. Guo, and J. Zhu, "System-level robust design optimization of a switched reluctance motor drive system considering multiple driving cycles," IEEE Transactions on Energy Conversion, vol. 36, no. 1, pp. 348-357, 2021.

[30] M. C. Pavkovic and P. Danilo, "Frequency-modulated pulsejet control of an artillery rocket," Journal of Spacecraft and Rockets, vol. 49, 2012. 